\title{
Sulla voce ne La nota segreta di Marta Morazzoni
}

\section{On the voice in La nota segreta by Marta Morazzoni}

\author{
Barbara Kornacka \\ Università Adam Mickiewicz di Poznań \\ kornacka@amu.edu.pl
}

\begin{abstract}
The aim of the article is to analyze the meanings of the voice in the novel mentioned in the title in relation to Adriana Cavarero's thought. Following the presentation of the work, the article examines two different manifestations of the voice in the text: the singing voice and the discursive voice. The former confirms the patriarchal association of women with the body but sometimes it also appears as a space of freedom from oppression. The latter, which is denied to the protagonist, symbolizes women's exclusion from logos.
\end{abstract}

Keywords: Marta Morazzoni, voice, women, Italian literature malmonacata

\section{MARTA MORAZZONI}

Che la prosa di Marta Morazzoni incanti, lo sanno tutti i lettori della raffinata scrittura di questa scrittrice. Al contempo traduttrice e critico teatrale, Morazzoni (classe '50) dal $1986^{1}$, anno di pubblicazione della sua raccolta d'esordio La ragazza col turbante, ci ammalia con le qualità delle sue opere: emozioni sottilmente allestite, elegante linguaggio, maestrali immagini nelle quali porta il lettore. L'autrice con grande sapienza ci conduce spesso nei tempi remoti e nei luoghi che pur essendo conosciuti, paiono estranei. Ogni volta sembra che si tratti dell'"invenzione della verità" ${ }^{2}$ : ossimoro che definisce meglio la sua prosa.

\footnotetext{
${ }^{1}$ Per le informazioni bio-bibliografiche vedi Kornacka, 2017.

2 Titolo dell'opera di M. Morazzoni, 1888, Invenzione della verità, Milano: Longanesi.
} 
La nota segreta, libro uscito nel 2010, cui si vogliono dedicare le presenti riflessioni, è un romanzo che non si sottrae a questa riassuntiva caratteristica della sua narrativa. Morazzoni riesuma dai meandri della Storia, per poi ricostruire e animare la storia di Paola Teresa Pietra, principessa milanese nel Settecento, realmente esistita $^{3}$, costretta dal padre a diventare monaca, motivo piuttosto ricorrente nella letteratura italiana, cosa cui torneremo a tempo debito. La vicenda di Paola è narrata attraverso il prisma di un particolare pertinente per la storia della donna e cruciale per più motivi per questa analisi, ovvero il suo straordinario talento vocale.

Quello che ci interessa anzi in questa sede è l'attenzione che l'autrice presta alla vocalità della protagonista e alla voce in generale nonché il motivo per cui lo fa. All'avviso di chi scrive il tema della voce nel romanzo non è né casuale né circostanziale bensì Morazzoni lo espone nella storia di Paola per i vari significati filosofici e metaforici che comporta e, di conseguenza, per il messaggio che veicola nel testo. Anche il titolo del romanzo, tra l'altro, allude al suono e al canto. Lo scopo quindi delle presenti riflessioni è di analizzare la vocalità di una donna che si manifesta nel romanzo in primo luogo nel canto e in secondo nella voce discorsiva. Si cercherà di esporre i diversi contenuti di entrambi gli aspetti della voce: il senso filosofico e la potenzialità della voce canora nonché il "mutismo" della voce discorsiva.

Prima però di passare all'analisi incentrata sul tema della voce si devono fare alcune considerazioni sull'opera stessa.

\section{STRUTTURA, TRAMA, PROTAGONISTA}

I cinque capitoli in cui si articola la struttura del romanzo corrispondono a cinque luoghi - Milano, La Serenissima, Mediterraneo, Le Cinque Querce, Roma - in cui si svolge la storia di Paola. Inoltre, coincidono approssimativamente con cinque fasi temporali identificabili come: cinque anni di adolescenza monacale; due settimane di aprile passate con John Breval a Venezia dopo la fuga dal convento; tre settimane di viaggio solitario a bordo di una nave mercantile da Venezia a Marsiglia; circa dieci mesi del soggiorno nella casa di Breval in Inghilterra fino all'aprile dell'anno successivo; alcune settimane che precedono e seguono il viaggio a Roma inclusa l'attesa del processo. In questi periodi e nei luoghi indicati si possono poi individuare cinque "stadi di mutazione interiore e esteriore" (234) della protagonista. $\mathrm{Si}$ potrebbe definirli come ribellione adolescenziale e chiusura forzata (Milano), scoperta dell'amore carnale e della femminilità del proprio corpo (La Serenissima), scoperta del mondo e della libertà nel senso lato del termine (Mediterraneo),

\footnotetext{
${ }^{3}$ Nella Nota dell'autrice Morazzoni indica le fonti di cui si ispirava, tra cui uno studio storico su Paola Teresa Pietra. Cf. Vismara, 1991.
} 
spaesamento e difficile ambientazione in Inghilterra come donna del mondo (Le Sette Querce), maternità, maturità e lotta per la propria libertà (Roma).

Da queste poche considerazioni sull'organizzazione interna del romanzo si desume in parte la trama che si potrebbe riassumere in maniera seguente. Paola Teresa Pietra, figlia di un principe milanese a tredici anni è costretta dal padre alla clausura monacale. Unica gioia, svago e soddisfazione è il canto, per il quale la ragazza si scopre meravigliosamente portata. Un giorno, durante una messa funebre Paola soffre di un mancamento. Svenuta, viene portata in sagrestia da un diplomatico inglese in missione a Milano in quel tempo e visitatore casuale della chiesa. John Breval è un uomo maturo, marito e padre di due figli, il quale però rimane particolarmente suggestionato dal viso della ragazza che vede in quel momento e, scoperte in seguito le sue capacità canore, si appassiona a lei. Anche nella memoria dell'oramai diciottenne monaca vive un vago ricordo olfattivo dell'uomo il quale rafforza la convinzione maturata nella donna di non voler più continuare la vita in clausura. John l'aiuta a fuggire e la porta a Venezia dove i due diventano amanti. Da lì però le loro strade si separano: John torna a Milano per completare i suoi incarichi diplomatici e la ragazza si imbarca su una nave mercantile che la deve portare secondo la promessa del capitano data a Breval - a Marsiglia decorsi 15 giorni, dove l'aspetterà John. Insieme a lui, tuttavia, l'aspettano gli uomini dell'arciduca milanese e gli emissari di Santa Chiesa Romana, tutti con l'intenzione di riportarla al convento. Nel viaggio per mare Paula scopre di non avere alcuna nozione geografica e rimane impressionata dall'immensità dello spazio aperto e dalla vastità del mondo nonché dalla strana libertà che le dà il travestimento da marinaio. La nave, tre giorni prima dell'approdo è attaccata e saccheggiata dai pirati per cui arriva a Marsiglia una settimana dopo il termine previsto. L'aspetta oramai solo l'amante non scoraggiato dalle voci secondo le quali la nave avrebbe fatto naufragio. John porta Paula alla sua casa famigliare in Inghilterra, dove vivono le sue sorelle minori. Per entrambi la situazione è difficile: lui chiede il divorzio a sua moglie e rinuncia al prestigioso lavoro di diplomatico, lei ha molte difficoltà ad ambientarsi nella nuova realtà e a comunicare con le sorelle di John. Dopo breve tempo scopre di essere incinta. Poco prima della nascita di Francesco, arriva la lettera dalla Penitenzieria Romana. Paola decide di affrontare la Chiesa per chiedere di essere ufficialmente liberata dai voti che non ha scelto per sua volontà.

Di figure di monache forzate la letteratura italiana possiede un repertorio piuttosto ricco, a cominciare dall'esempio più canonico, per non dire iconico ${ }^{4}$, della mo-

\footnotetext{
${ }^{4}$ La famosa Gertrude ossia Marianna de Leyva è oramai oggetto non solo di studi letterari e semiotici (Calabrese, 1989), ma anche di opere d'arte dall'ottocento in poi, di cui racconta la mostra tenuta a Monza nel Serrone della Villa Reale della Regia (cf. Una mostra che racconta la vera storia della celebre monaca di Monza, http:/www.arte.rai.it/articoli/una-mostra-racconta-la-vera-storia-dellacelebre-monaca-di-monza/35466/default.aspx), nonché di parodie e travestimenti nella cultura di massa contemporanea a cominciare da Guido da Verona (da Verona, 1930).
} 
naca di Monza manzoniana, alla cui fama letteraria Morazzoni allude ironicamente nel momento di presentare la sua protagonista:

In quanti, a questo punto, staranno pensando che mi aleggia attorno un tale illustre precedente, che farei meglio a demordere per evitale un confronto a me fatale! Ma ho il vantaggio di sentirmi tanto al disotto del termine di paragone, da non avere remore a espormi. Come dire? perso per perso, tant'è giocare la partita fino in fondo (11).

Nella letteratura italiana, soprattutto quella ottocentesca, il tema della monacazione forzata ritorna più volte. Gli esempi più conosciuti sono Ildegonda di Tommaso Grossi (1820), Il Monastero di Sambucina di Vincenzo Padula (1842), Estella di Arnaldo Fucinato (1853), La suora di Luigi Carrer (1854), Storia di una capinera di Giovanni Verga (1869) ${ }^{5}$, Giambattista Pergolesi di Francesco Mastriani (1874). Volendo ora indicare qualche genealogia letteraria del romanzo di Morazzoni, pare invece più fondato accostare la figura di Paola alle voci di Arcangela Tarabotti (cfr. Contu, 2016, 120-142) e di Enrichetta Caracciolo (cfr. Miszalska, 2017, 320), anche loro figure documentate e realmente esistite, rispettivamente nel Seicento e nell'Ottocento, perché entrambe cercarono di ribellarsi e di lottare contro il sistema patriarcale ricorrendo alla scrittura. Similmente Paola, per opporsi alla Chiesa Cattolica, una delle istituzioni più potenti della società patriarcale, e quindi per difendere il diritto alla propria vita, usa infine la scrittura ${ }^{6}$.

\section{VOCE}

Nel romanzo analizzato, sotto la superficie della coinvolgente narrazione delle vicende, talvolta rocambolesche, talvolta romantiche, della giovane principessamonaca Paola Teresa Pietra, pare avere il suo corso sottocutaneo, un altro racconto. Ad afferrare le sue tracce e a seguirlo ci invita la parola che forse ritorna più frequentemente nel testo - la voce - sin dalla prima pagina, dove le monache sono definite come "compagne di vocazione: chiamate dalla voce di Dio". Questo gioco di termini - vocazione, voce di Dio - annuncia l'intrecciarsi della vicenda narrata della monaca forzata (livello della trama) con la tematizzazione della voce nel romanzo (livello del pensiero filosofico), cominciando proprio dal riferimento "alla sfera acustica della presenza del divino" (Cavarero, 2010, 27). Quest'ultima, infatti, come dice Cavarero, sta all'origine di molte culture (Cavarero, 2010, 27).

\footnotetext{
${ }^{5}$ A proposto del confronto tra i personaggi di Verga e di Matilde Serao Suor Giovanna della Croce (1899) vedi Miszalska, 2017, 318-334.

${ }^{6}$ È naturale qui pensare alla scrittura come strumento di riscatto e di lotta delle donne nel corso della storia, tema metaforizzato ad esempio da Dacia Maraini nella sua famosa opera La lunga vita di Marianna Ucrìa, argomento cui si tornerà più tardi.
} 
La voce, sia come una metafora versatile sia nel suo senso letterale, ovvero la più svariata produzione vocale acustica, fa parte della letteratura e come tale è studiata ${ }^{7}$. Nel pensiero femminista la voce diventa metafora di un vasto repertorio di aspirazioni delle donne: diritti politici, rappresentanza nella cultura, indipendenza sessuale, libertà di espressione e in quest'ultimo contesto la voce metaforizza il potere del testo, della scrittura (Dunn, Jones, 1996, 1). Il termine "voce" ha inoltre la sua ampia storia nella riflessione filosofica - coinvolgendo la metafisica, l'ontologia e la fenomenologa - da Socrate a Barthes e Derrida (cfr. Michalik, 2010; Cavarero, 2010). Come scrive Joanna Michalik sin dalle origini dell'interesse che la filosofia ebbe per la voce, essa s'iscriveva nella costellazione di nozioni metafisiche: nella triade dell'essere, del pensiero e dell'espressione (11).

Per le analisi che seguono si farà soprattutto ricorso alle riflessioni e alle ricerche della filosofa italiana Adriana Cavarero nonché alla sua teoria della devocalizzazione del logos. Come osserva la studiosa, la voce nella cultura classica greca veniva accomunata nell'orizzonte acustico a tutti gli altri suoni, anche quelli animaleschi, e inglobata nel termine phonè, tendendo l'acustico in questo modo a costruire una sfera autonoma da quella della parola (Cavarero, 2010, 27). Infatti, come spiega in seguito la filosofa, anche se Aristotele definisce il logos come phonè semantikè (Aristotele) $)^{8}$, voce significante, è il secondo termine a prevalere nel logos mentre il primo serve solo da supporto acustico, non essendo neanche necessario. Questa predominanza del semantikè sul phonè si fa chiara se si considera come "elemento fondante del logos il nesso della parola con il regime dei significati" (Cavarero, 2010, 45) o se si riporta il logos al mondo delle idee, più ancorate nella sfera del visivo che nella sfera dell'acustico (Cavarero, 2010, 51-52). Così si giunge alla devocalizzazione del logos, strategia in cui la voce (parte acustica) diventa superflua e contaminante la parola, essendo un filtro che ostacola l'accesso alla verità. Invece il logos, pienamente identificato con la parte semantica della parola, rimane un mondo di significati insonori e visibili che vuole coincidere il più possibile con il pensiero (Cavarero, 2010, 53-54) ${ }^{9}$.

All'avviso di chi scrive il romanzo di Morazzoni nello strato di riflessione filosofica parla appunto del processo di devocalizzazione del logos, dell'avvenuta scissione tra la voce (phonè) e il pensiero, il senso e la ragione (semantikon che equivale quindi al logos) ovvero della privazione discriminante della voce della sua parte

\footnotetext{
${ }^{7}$ Nella letteratura italiana opere che tematizzano la voce e che al tempo stesso costituiscono un contributo alla riflessione filosofica sulla voce sono il racconto di Calvino Il re in ascolto, e Voci di Dacia Maraini (cf. Kornacka, 2006). Circa lo studio della voce nella letteratura cf. Dunn, Jones, 1996.

${ }^{8}$ Citato da Cavarero, 2010, 43.

${ }^{9}$ Cavarero nell'ultimo capitolo della sua opera critica apertamente il sistema fonologocentrico proposto da Derrida spiegando che tutta la sua ricerca sulla devocalizzazione del logos parte dalle posizioni anti-derridiane (233-263).
} 
attiva nella produzione del senso e della verità, attribuendo a entrambe le parti un'identificazione di genere: femminile per la vocalità, maschile per il pensiero.

Cercherò di dimostrarlo analizzando due aspetti della voce presenti nel libro ovvero il canto e la voce discorsiva.

\subsection{Canto}

Analizzando il canto che - sia per Paola stessa sia nell'economia del romanzo svolge un ruolo di primo piano, si arriva a uno stereotipo di lunghissima data, diventato nell'ordine simbolico patriarcale pressoché un apriori, quello che collega il femminile al corporeo e il maschile al razionale, ovvero il femminile al vocale e il maschile al semantico. Per dirla brevemente con Cavarero: "La donna canta, l'uomo pensa" (Cavarero, 2010, 12). Infatti, il canto nel romanzo di Morazzoni, soprattutto quello della protagonista, crea alcune associazioni che portano alle antiche Sirene, non quelle di Omero, narratrici onniscienti (Cavarero, 2010, 119-120), ma quelle che sono diventate nel secolare sistema androcentrico: donne-mostri, donne-uccelli, donne-pesci - queste ultime bellissime e sensuali - che con il loro canto armonioso, voce bellissima e sensuale ammagliano e seducono gli uomini, portandogli la morte. Le Sirene cantano ma non hanno la capacità di raccontare, non dicono niente, la loro è una pura voce, spoglia della parte semantica, pura phonè. Esse illustrano e confermano la dicotomia dell'ordine simbolico patriarcale che vede "lo scindersi del logos in pura phonè femminile e in puro semantikon maschile" (Cavarero, 2010, 119).

Suor Paola che incanta con il canto senza essere vista, incarna molti aspetti dell'immagine della Sirena, e non solo perché, come dice Cavarero: "la donna che canta è sempre una sirena, ossia una creatura dell'ordine del godimento, estranea all'ordine domestico di figlie e spose" ${ }^{, 10}$ (Cavarero, 2010, 131), come lo sono anche le monache. Tra sirene e monache - sorprendentemente - vi corrono più parallelismi, a cominciare dalla sensualità dello stesso cantare, potere eccitante, quasi erotico del canto, "veicolo subdolo della seduzione" (37) come lo vedeva la badessa molto contraria alle prestazioni canore nel convento. Le temeva per la loro "ricaduta mondana" (18), provando anche dentro di sé un'eccitazione sensuale provocata dal canto: "Le era molto d'imbarazzo dare il nome giusto alla sensazione che avvertiva e al solletico che faceva anche a lei, un prurito sensuale, ma qui la madre badessa si fermava sulla soglia della parola temuta" (38). A subire l'effetto più esplicito, inevitabile e in qualche modo anche distruttivo del canto seducente di Paola è un uomo, John Breval, attratto sempre di più dalla voce della giovane monaca e inte-

${ }^{10}$ Infatti anche questo dettaglio coincide con la storia di Paola. Nel romanzo la protagonista canta l'ultima volta e oramai a bassa voce, quando si rende conto di essere incinta. Dalla danna ammaliatrice, donna dell'ordine di godimento, diventa donna dell'ordine domestico. 
ressato sempre di più alla sua persona. Mentre la sente cantare lo Stabat Mater ${ }^{11}$ di Pergolesi da dietro la grata diventa particolarmente "agitato e inquieto" (39), il "non poter vedere nulla accendeva la fantasia di una sequenza di immagini che lo agitavano e lo appaga a un tempo" (39) e quando "lo Stabat Mater finì [che] sir John era perso in una fantasia erotica conturbante" (40). L'effetto seducente del canto ha qualcosa di magico, perché continua una volta finita l'esecuzione. John "si svegliava di notte con in mente la voce profonda del contralto nello Stabat Mater e ne accarezzava il timbro come avrebbe fatto della pelle di lei [...]. La mente lo portava più del dovuto verso il convento, più del voluto anche, perché finì per farne spesso argomento di conversazione con i convitati alle tante cene cui si trovava a partecipare" (43-44). Paola non è veramente una Sirena quindi il suo canto non produce effetti veramente letali, tuttavia la passione e l'innamoramento che ne sono frutto porteranno John Breval (viaggiatore in terre straniere in questo caso) a sconvolgere la propria vita: divorzio e dimissione da un incarico molto prestigioso. Sarà lui stesso a pensare: "[...] quanto peso aveva avuto nel suo innamorarsi di una sconosciuta la seduzione della voce" (220). Invece ciò che accomuna il canto di Paola a quello delle Sirene è la mancanza di parole, la pura phonè. Non vengono mai menzionati i contenuti dei canti, le parole cantate. Anzi, sono sia Paola sia suor Rosalba a sottolineare che non si dà alcuna "attenzione alle parole" (40). L'opera nominata più volte nel testo, eseguita più spesso è lo Stabat Mater di Pergolesi di cui non vengono mai riportate, commentate o interpretate le parole, scritte da Jacopone da Todi. La preghiera a Paola "piacque molto e non si curò affatto delle parole, ci pensava la musica a marcare tragedia e elegia" (37). Ebbene, nel canto è la musica ad assumere il significato delle parole, è la musica a ridare alla pura phonè la possibilità di significare, anche senza ricorso alla sfera razionale, senza parole. È un meccanismo simile a quello che si attua nell'opera, dove le parole non vengono spesso capite per motivo della lingua o della pronuncia, per cui "il canto prevale sul significato, la voce sulle parole, il vocalico sul semantico, e la musica sulla storia. [...] Questa è 1'opera dell'opera: voci che vanificano il ruolo della parola. Un godibile trionfo del vocalico sul semantico" (Cavarero, 2010, 136).

Tuttavia Morazzoni, nonostante l'evidente prepotenza del semantikon nel generare il senso, cerca di fare significare anche la voce stessa, renderla non più solo un supporto o un ostacolo, ma di incaricarla di un significato autonomo. La scrittrice lungo tutto il romanzo descrive le qualità della voce della protagonista: traduce in registro grafico il registro sonoro. Quella della giovane Paola è quindi "una voce strana e scura" (15), "piena e profonda" (96), "un contralto di rara potenza. Una qualità virile appena ammorbidita da una inflessione più dolce" (16), è "come il suo stesso nome: solida e definitiva" (15). Cadendo nella trappola degli stereotipi di

\footnotetext{
${ }^{11}$ Secondo Kristeva lo Stabat Mater nasconde una particolare tensione sensuale (Kristeva, 1983, 311-327).
} 
genere vi si potrebbero vedere sfumature maschili (potenza, solidità, oscurità), un'eco di un carattere determinato, valoroso e coraggioso, da maschio. Infatti, tale si sarebbe rivelato poi il carattere della protagonista - ricorrendo sempre alle classificazioni stereotipate - ma libero tuttavia da questo prisma si è piuttosto propensi a capire il senso nascosto nelle qualità della voce di Paola come manifestazione della sua unicità ${ }^{12}$ oppure come sintomo della sua soggettività che si situa tra due estre mità di concepire la natura umana, definita oltretutto in quanto razionalità $\mathrm{o}$ in quanto affettività (Michalik, 2010, 118).

Nel romanzo analizzato, poi, si osserva un ancora altro aspetto del canto, sempre in sintonia con le considerazioni sul godimento e sulla seduzione che deriva da questa attività, sulla passione e sul corpo che lo producono, ma che tuttavia porta a conclusioni diverse. Suor Paola, "sepolta viva" (13) nel convento, non ha la vocazione, ovvero la voce della chiamata di Dio, non l'ha mai sentita. Dall'inizio della sua clausura sembra invece essere molto attratta dalla voce delle suore coriste. Percepiva il loro canto come l'unico filo che collegava il convento con il mondo di fuori: "Cominciò a meditare sulla forza d'attrazione del canto fino a essere punta dall'invidia per le voci che catturavano tanta attenzione: di là della grata c'era il mondo attaccato per un filo alle sepolte vive di cui lei era parte" (12-13). Appena scoperto il suo singolare talento vocale, potrà anche lei godere quel piacere corporeo: "era il suo corpo che produceva la melodia e la alzava, la smorzava, vibrava di una passione che lei stessa non si sarebbe altrimenti mai riconosciuta" (40), piacere in cui "natura e cultura richiamavano la loro parte" (16), scoprendo la propria vocazione che "confondeva la religione con la musica" (20). Il canto la seduce e presto diventa per lei "una ragione di vita quale mai si sarebbe potuta aspettare nella casa scura di suo padre, nel mondo degli aristocratici che aveva appena fatto in tempo a conoscere, e meno che mai accanto a un potenziale marito" (19). Nella rigida clausura dell'ordine delle benedettine, più severa di ogni altra istituzione dell'ordine patriarcale, dove le monache vivono una condizione di totale assoggettamento, di privazione della loro soggettività e di negazione della loro corporeità, il canto diventa uno spazio di libertà che le seduce, in primo luogo loro stesse, diventa l'unica possibilità di espressione soggettiva e di piacere corporeo, altrimenti ammutoliti. Il canto, da questa prospettiva, lo si può considerare come una metaforica invocazione dei diritti negati, tra cui il diritto al logos, all'espressione del pensiero e il diritto all'unicità.

Orbene, mentre Paola canta: dispone di una voce ammaliante ma spoglia della parte semantica, o quasi, una pura phonè, John risulta pienamente depositario delle

\footnotetext{
${ }^{12}$ Riferimento alle parole di Cavarero sulla verità del vocalico: "la quale, lungi dall'essere astratta come le verità poste dalla ragione, proclama semplicemente che ogni essere umano è un essere unico ed è capace di manifestarlo con la voce, chiamando e contagiando l'altro, e godendo, per di più, di tale reciproca manifestazione" (Cavarero, 2010, 13).
} 
facoltà che dà il logos. Con ciò tuttavia passiamo al secondo aspetto della riflessione sulla voce presente tra le righe del romanzo ovvero alla voce discorsiva vale a dire alla voce (phonè) che pur ausiliare nella produzione del senso, pur essendo un puro veicolo del semantikon, nella produzione orale è necessaria per comunicare la parola, il pensiero, il senso.

\subsection{Voce discorsiva}

John, controparte maschile della figura femminile nella struttura del romanzo, è un tipico soggetto razionale e logocentrico, un Ulisse che però non torna alla sua Penelope bensì si lascia incantare dalla voce di una Sirena. Tale sarebbe l'analogia che si muove sulla stessa scia. John ha eccellenti doti comunicative: è diplomatico, conosce diverse lingue, padroneggia alla perfezione l'arte della conversazione e della trattativa, parla con destrezza con l'arciduca di Milano, con il doge di Venezia, con la badessa del convento di Santa Radegonda. Inoltre, John comunica con sua moglie e le sue sorelle scrivendo numerose lettere ed è lui che progetta la fuga di Paola, escogita piani che richiedono astuzia. Insomma, ha le capacità che confermano la sua "mente lucida e aperta" (69) o, in altre parole, la sua appartenenza alla sfera del logos.

Paola invece non parla, o parla pochissimo, quasi a comprovare la convinzione vigente nell'ordine patriarcale che alle donne si addice il silenzio. In maniera sorprendente Morazzoni costruisce questo pressoché totale mutismo della protagonista. Nel romanzo ci sono poche scene in cui leggiamo le parole pronunciate dalla protagonista, ovvero la "sentiamo" parlare. La sua voce discorsiva, al contrario di quella di John, è molto modesta e limitata allo stretto necessario. Le frasi pronunciate da lei sono brevi e sintetiche, a cominciare dalla prima, sintomatica, rivolta alla sua maestra del canto: "Lo canteremo ancora?" riferita allo Stabat Mater, che appare solo alla quarantaduesima pagina. Sono invece piuttosto frequenti i riferimenti al silenzio e al mutismo. Nel convento, come narra Morazzoni, non avendo amiche o persone con cui parlare "Paola visse in una condizione di mutismo, sepolta dentro se stessa" (59). Con John, con cui la storia d'amore parte dall'incanto della voce e dalle sensazioni olfattive nascoste nella memoria di lei, inizialmente non hanno molto da dirsi: "non hanno parole per dirsi alcunché" (68) e invece di ricorrere alle parole per conoscersi lasciano che i corpi continuino a farlo: gesti, baci, carezze, amore sono accompagnati dal silenzio. "Rimasero l'uno addosso all'altra muti [...]" (87) scrive l'autrice oppure, in un altro momento leggiamo: "Si erano detti, lo abbiamo sentito, ben poche parole, e ora il silenzio si fece ancora più esteso nella stanza dentro cui passava la luce dell'ultimo sole" (167). Mentre con l'amante è il corpo quasi a sostituire la voce discorsiva, essa si dimostra uno strumento totalmente inefficace in Francia o in Inghilterra per la sconoscenza delle lingue. Lo esprime la 
donna stessa: "Non potrò dire niente con le tue sorelle, se non ci sarai tu. A lungo non potrò dire niente. Come una muta, osservò uscendo dal lungo silenzio" (191). Infatti, appena arrivata a casa delle sorelle di John, ha luogo una scena costruita "dai minuti dettagli di piccoli passi, di gesti di cortesia, che sono tramite di un linguaggio senza parole nel quale si inquadra la svolta da imprimere alla storia di Paola Pietra" (198). Non capendo la lingua Paola si sente non solo muta, ma anche sorda, per cui esclusa, estraniata. Inutilmente cercherà di abbattere questa barriera con la bellezza della sua phonè, con "la bella voce di contralto che modulò senza affettazione" (201). La sua voce discorsiva si dimostra nella casa de "Le sette querce" non funzionante. Paola è di nuovo condannata alla condizione di mutismo tanto più dolente che questa volta le è stato tolto anche il piacere - e quindi la libertà - del canto.

La sfida più importante posta davanti alla sua voce sarà tuttavia quella di parlare alla Penitenzieria della Chiesa Romana per chiedere di essere liberata dai voti, per farsi ascoltare e per fare ascoltare le sue ragioni, un compito non di poco conto data l'oggettivazione della donna (tanto più di una monaca fuggiasca) nell'ordine patriarcale. Tuttavia neanche questa volta la "sentiamo" parlare. Il lettore fino all'ultima pagina del romanzo aspetta questo momento: Paola che finalmente fa il discorso più importante della sua vita, il discorso che vale la sua libertà: esprime i suoi pensieri, le ragioni, gli argomenti per difendere i suoi diritti. E invece la scena finale del romanzo chiudendosi con le parole: "[...] poi formalmente diede la parola a Paola e ascoltò" (273), non riporta le parole di Paola. Anche in questa circostanza le è stata tolta la voce, soluzione che rileva un'ennesima volta la condizione del mutismo della donna. Per di più, anche se la protagonista in quel momento parlasse, il suo discorso non avrebbe alcuna importanza, essendo la sentenza già pronta prima di ascoltarlo. "Nella mente del cardinal Petra la giovane nobile milanese Pietra, figlia del conte Francesco Brunerio Pietra, era già assolta da ogni peccato, sciolta dal voto e mandata libera" (268). È singolare che al giudizio del prelato non contribuisca in alcuna misura quello che ha da dire la donna ossia la sua voce discorsiva, bensì esso si basa unicamente sui documenti raccolti ovvero sulla parola scritta, tra cui la lettera di Paula alla Penitenzieria della Chiesa Romana. Se ne evince che solo ricorrendo alla scrittura la donna riesce a fare sentire le proprie ragioni, solo per mezzo della scrittura, strumento del logos devocalizzato, può affrontare il sistema.

S'impone con naturalezza e spontaneità un'altra analogia letteraria: quella con il personaggio di Marianna Ucrìa de La lunga vita di Marianna Ucrì di Dacia Maraini. Analoghe sono non solo l'ambientazione storica di entrambi i romanzi, l'estrazione sociale delle protagoniste e la loro infanzia traumatica ma anche l'importanza della voce per ambedue le donne. Marianna perde la voce ${ }^{13}$ o la rifiuta

${ }^{13}$ Tolta la voce, strumento del logos, le facoltà intellettive di Marianna sono ampliate - la comprensione del mondo circostante - dall'uso di altri sensi, in particolare dall'olfatto, a rivalutare e ribadire la partecipazione dell'intero corpo alla comprensione della realtà. Cf. Kornacka, 2007, 201-216. 
in atto di ribellione dopo la violenza subita, Paola in parte acquisisce la voce quella canora - proprio grazie alla clausura quindi grazie al trauma subito e, in parte - se si tratta della voce discorsiva - non la utilizza mai. Analoga è inoltre la metaforizzazione della voce dietro alla quale si cela il discorso sulla privazione delle donne dei diritti personali, sociali e politici. Per Paola, la voce canora, come si è detto, costituisce inoltre la possibilità trasgredire la condanna al silenzio, possibilità che invece Marianna ritrova nella biblioteca e nell'istruzione. Analogo infine è anche il ricorso alla scrittura come unica strategia per riconquistare la libertà personale e almeno una parte dei diritti soppressi.

\section{CONCLUSIONI}

Il romanzo analizzato nel suo strato puramente narrativo rievoca la vicenda di una malmonacata del Seicento milanese, una tra le tante nella storia della Chiesa e nella letteratura italiana, che si ribella contro la vita, impostale dagli altri, contro il sacrificio non voluto. Visto come tale, come un racconto, è un romanzo sicuramente molto istruttivo e commovente che ci parla del coraggio femminile e della potenza del desiderio di essere libere. Sarebbe tuttavia uno sguardo riduttivo sul libro preso in esame. Alla sua pienezza si giunge solo chinandosi anche sullo strato di riflessione filosofica sulla voce che la scrittrice sapientemente intreccia tra le righe del testo e tra le svolte narrative. Da essa si desume che la voce canora è sinonimo del godimento, ma non è portatrice del semantico $^{14}$, e riportata all'immagine della donna che canta sta a confermare lo stereotipo vigente nella cultura patriarcale e ricostruito abilmente dall'autrice, lo stereotipo che colloca il femminile nella sfera del corporeo, cosa accentuata inoltre dai numerosi riferimenti al corpo, anche nudo, di Paola. Tuttavia nel canto si nasconde anche un potere trasgressivo essendo uno strumento di piacere e di libera espressione della propria unicità, laddove, come nella clausura conventuale, l'oppressione è totale. Invece, la riflessione sulla voce discorsiva, sul modo in cui viene costruito da Morazzoni il pressoché totale mutismo della protagonista - che tace o non può effettivamente comunicare per mezzo della voce, necessaria alla produzione del semantico - ci induce a vedervi la negazione della voce alla donna. Con questa strategia l'autrice allude alla negazione dei diritti alla donna nel corso dei secoli nella cultura patriarcale rappresentata nel romanzo dal padre, dalla chiesa ed anche, in ultimo luogo, dall'amante. Infatti, la negazione della voce discorsiva preclude alla donna l'accesso alla sfera del razionale ovvero alla sfera del logos. Soltanto ricorrendo alla parola scritta la donna può sperare di riuscire a raggirare i modelli stereotipati della cultura patriarcale e fare riconosce il suo diritto come depositaria paritaria del logos e vincere la sua causa.

\footnotetext{
${ }^{14}$ Da lì l'accezione dispregiativa dell'aggettivo "canoro" ovvero "vuoto, superficialmente retorico" . Cf. Lo Zingarelli, 2017.
} 


\section{BIBLIOGRAFIA}

Calabrese, O. (1989). L'iconologia della monaca di Monza. In G. Mannetti (Ed.), Leggere i Promessi sposi: analisi semiotica. Milano: Fabbri - Bompiani - Sonzogno.

Cannelli, M., Lazzerini, B. (Eds.). (2017). Vocabolario della lingua italiana di Nicola Zingarelli. Bolonia: Zanichelli.

Cavarero, A. (2010). A più voci. Filosofia dell'espressione vocale. Milano: Feltrinelli.

Contu, F. (2016). Teatro e monacazione forzata in Italia tra Cinque e Seicento: tre figure emblematiche. In M. Galletti, F. Contu, Fuori controllo. Donne e teatro tra Cinque e Seicento in Italia (pp. 65-171). Sevilla: Benilde Ediciones.

Dunn, L.C., Jones, N.A. (1996). Introduction. In L.C. Dunn, N.A. Jones, Embodied Voices: Representing Female Vocality in Western Culture. Cambridge: Cambridge University Press.

Kornacka, B. (2006). La scrittura udibile - alcune osservazioni sul romanzo Voci di Dacia Maraini. Écho des études romanes. Revue semestrielle de linguistique et littératures romanes, vol. II, n. 2, 71-87.

Kornacka, B. (2007). Un silenzio molto profumato - alcune riflessioni sul romanzo La lunga vita di Marianna Ucrì di Dacia Maraini. Studia Romanica Posnaniensia, XXXIV, 201-216.

Kornacka, B. (2017). Marta Morazzoni. Retrieved from https://modernlanguages.sas.ac.uk/researchcentres/centre-study-contemporary-womens-writing/languages/italian/marta-morazzoni

Kristeva, J. (1983). Histoires d'amour. Paris: Denoël.

Michalik, J. (2010). Filozofia i głos. Kraków: Nomos.

Miszalska, J. (2017). La figura della monaca: due sguardi a confronto. Storia di una capinera di Giovanni Verga e Suor Giovanna della Croce di Matilde Serao. In A.S. Villarroya (Ed.), Personajes femeninos y Canon, 11-13 dicembre 2017 (pp. 318-334). Sevilla: Benilde Ediciones. Retrieved from https://idus.us.es/xmlui/handle/11441/68170

Morazzoni, M. (2012). La nota segreta. Milano: TEA.

da Verona, G. (1930). I promessi sposi. Milano: Unitas.

Vismara, P. (1991). Per vim et metum. Il caso di Paola Teresa Pietra. Como: Litografia New Press. 